\title{
The Mental Health of People with Intellectual Disabilities who Offend
}

\author{
B. Hobson and J.L. Rose*
}

School of Psychology, The University of Birmingham, Edgbaston, Birmingham, B15 2TT, UK

\begin{abstract}
There are a growing number of studies concerning people with intellectually disabilities (ID) who offend, however few studies systematically record prevalence of mental health problems. This article will attempt to determine the prevalence mental health problems in people with ID who offend. Search terms were entered into the CINAHL, EMBASE, Ovid MEDLINE(R) and PsycINFO databases. Studies were also selected through discussion with clinicians and references from relevant papers. Fourteen relevant articles were found that indicated the prevalence of mental health problems in people with an ID who offend. Mental health problems may be more prevalent in individuals with an ID who offend than those with an ID who do not offend with schizophrenia/psychotic difficulties occurring more frequently. The possible increased prevalence should be considered within treatment regimes in order to effectively rehabilitate individuals.
\end{abstract}

\section{INTRODUCTION}

A number of reports have attempted to describe people with an intellectual disability (ID) who offend $[1,2]$ however only a minority of these have considered the influence of mental health problems. People with an ID who offend and have mental health difficulties may be triply stigmatized with the labels of "criminal", "disabled" and "psychiatric" with services for these individuals being neglected [3].

There are three core criteria for the definition of intellectual disability: These include 1). Significant impairment of intellectual functioning. 2). Significant impairment of adaptive/social functioning and 3). Age of onset before adulthood. All three criteria must be met for a person to be considered to have an intellectual disability (e.g. British Psychological Society, 2001) [4]. General prevalence rates of mild intellectual disabilities across all ages have been shown to range between 3.7 and 5.9 per 1,000, with total population studies reporting higher rates than administrative samples from groups of people known to have an intellectual disability [5]. Some authors have suggested (e.g. Simpson and Hogg, 2001) [2] that there is no clear evidence of higher prevalence rates of forensic activity within the ID community as a whole, and offending in individuals with a severe learning disability is rare. However, sexual offending, criminal damage, and burglary may be over represented in people who have a mild or borderline intellectual disability, with crimes such as murder and armed robbery being underrepresented. Lindsay (2002) [6] suggests that the lack of control groups to compare offender populations, makes it difficult to determine the specific characteristics associated with offending in the ID population.

The mental health needs of people with an ID may go unmet until they commit a serious offence [7]. It has also been suggested that mental health can play a role in the offending process in people with an ID [8]. However there is a

*Address correspondence to these authors at the School of Psychology, The University of Birmingham, Edgbaston, Birmingham, B15 2TT, UK; Tel: 01214142640; Email: j.1.rose@bham.ac.uk paucity of studies regarding the prevalence of mental health issues in people with an ID who offend. Mental health problems may impact upon treatment and management of people with an ID who offend [9]. As a result it is of particular importance that we gain a clearer picture of how many individuals with an ID who offend also have mental health problems as this may impact on the efficacy of treatment and on rates of recidivism. This article will review the related literature in an attempt to reveal the prevalence in mental health problems in people with an ID who offend and make some tentative suggestions as to what implications this has for these individuals.

\section{METHOD}

The search terms shown in Table 1 were entered into the CINAHL, EMBASE, Ovid MEDLINE(R) and PsycINFO databases, with the following criteria applied:

- January 1990-October 2007

- English language

- Human

The terms used in the search were chosen from an inspection of the literature available. The date range was selected to include relatively recent literature. The terms are grouped into three areas and will be referred to as 'intellectual disability', 'mental health' and 'offending behavior'.

Duplicate papers were removed from the results and the identified articles were assessed in more detail for their relevance. Non-ID and non-adult populations were excluded. Finally, the reference sections of the remaining articles were scrutinized and expert clinicians consulted, in order to identify further relevant articles.

\section{RESULTS}

The search found a total of 405 articles, of which 393 were excluded leaving 12 that contained relevant data (Table 2). A further two studies were included after examining the references of studies and consulting clinicians; these were 
Table 1. Terms Used in the Search

\begin{tabular}{|c|c|c|}
\hline Intellectual Disability & Mental Health & Offending Behaviour \\
\hline \hline Developmental Disabilit* & Mental Health/Disorder/Illness & Offen* \\
\hline Intellectual Disabilit* & Pentally Ill & Police $*$ \\
\hline Learning Disabilit* & Psychiatric Disorder/Hospital/Nursing & Crim* \\
\hline Mental Handicap & Psychotic Disorders & \\
\hline Mental Retard* & & \\
\hline
\end{tabular}

Ho (1996) [10] and Rose, Cutler, Trezise, Novak and Rose (2008) [11].

Table 2. Criteria Used for the Exclusion of Articles

\begin{tabular}{|c|c|}
\hline Number of Studies & 405 \\
\hline \hline Criteria & $\begin{array}{c}\text { Number of Studies Ex- } \\
\text { cluded }\end{array}$ \\
\hline Non-adult & 37 \\
\hline Non-intellectual disability & 99 \\
\hline Non-offenders & 32 \\
\hline Non-mental health & 78 \\
\hline Non-empirical & 60 \\
\hline $\begin{array}{c}\text { No report of mental health problems in } \\
\text { people with an ID who offend }\end{array}$ & 87 \\
\hline Total Excluded Articles & 393 \\
\hline Additional papers identified & 2 \\
\hline Balance & 14 \\
\hline
\end{tabular}

The papers selected all reported rates of mental health problems in a range of samples of people with an intellectually disabled who offend. These rates are listed in Table 3.

Research reporting the prevalence of mental health problems in people with an ID who offend use three principal sampling strategies including surveys of individuals in criminal justice settings, community and in-patient populations.

\section{CRIMINAL JUSTICE SETTINGS}

Klimecki, Jenkinson and Wilson (1994) [12] surveyed a sample of individuals with an ID from a segregated prison population in Australia, the study identified seventy five individuals with an intellectual disability. All participants were reported to have an IQ in the range of 65 - 75 although a mean for the group is not reported, it was also not explicitly stated how IQ was assessed. Participant's psychiatric history was taken from prison records though it is not clear what criteria was used for the people with an ID to be segregated from the normal prison population, nor was the prevalence of ID stated within that population. An indication of having a "prior psychiatric history" was used to record whether individuals had mental heath problems in this group and it may have contributed to the high rate of mental health problems recorded $(74.5 \%)$. Klimecki et al. (1994) [12] found that rates of re-offending were higher in those individuals with a dual diagnosis of substance abuse and a prior psychiatric history.

Ho (1996) [10] conducted another retrospective study of case notes at a secure forensic hospital in Florida, USA, for those who were deemed incompetent to stand trial due to their level of intellectual ability. This sample was large (282) and IQ and adaptive behavior were assessed through psychological evaluation, competency reports and assessment using the WAIS-R and the Vineland Adaptive Behavior Scales. However, it is not clear who administered these tests nor is it evident when they were assessed. The mean IQ $(\mathrm{M}=58.0)$ was lower than in other studies, though a borderline group (4.6\% of the total sample) was also included (IQ scores between 70 and 85). The study group had a large (but not explicitly stated) IQ range. One individual was assessed by clinicians as having as IQ below 25, though it is unclear how many individuals were also assessed using clinical judgment rather than having an IQ assessment. Over half (52.3\%) of the sample was diagnosed or suspected of having a mental health problem, though it is not clear what criterion was used to make this assessment.

Winter, Holland and Collins (1997) [13] used a sample of individuals identified as having an ID while screened during police custody. The sample size was small (21) while the mean full-scale IQ from the WAIS-R assessment was 79.1 $(\mathrm{SD}=7.3)$, only two individuals in the study had an identified IQ score of below 70. As part of the screening process, individuals were asked if they had attended a school for children with ID, none of the study group had but five had attended a school for children with behavioral problems. The study also relied on participants self-reporting past medical and psychiatric history and drug and alcohol abuse, which may have led to the relatively low rates of mental health problems reported ( $15.8 \%$ psychosis, $11.1 \%$ affective disorders).

Crocker et al. (2007) [14] investigated a sample from a pre-trial detention centre in Canada. The IQ of individuals was assessed using the E'preuve Individuelle d'Habilete' Mentale (EIHM - Individual Mental Ability Scale), an intellectual ability scale standardized to the norms of the Quebec population. Using IQ scores, 281 individuals were assigned to two ID groups (probable and borderline) and no ID group. The mental health of participants was diagnosed using the Expertal expert system, which is based on DSM 4 criteria, with over half of the sample being re-assessed by a psy- 
Table 3. Prevalence of Mental Health Issues in Reviewed Articles

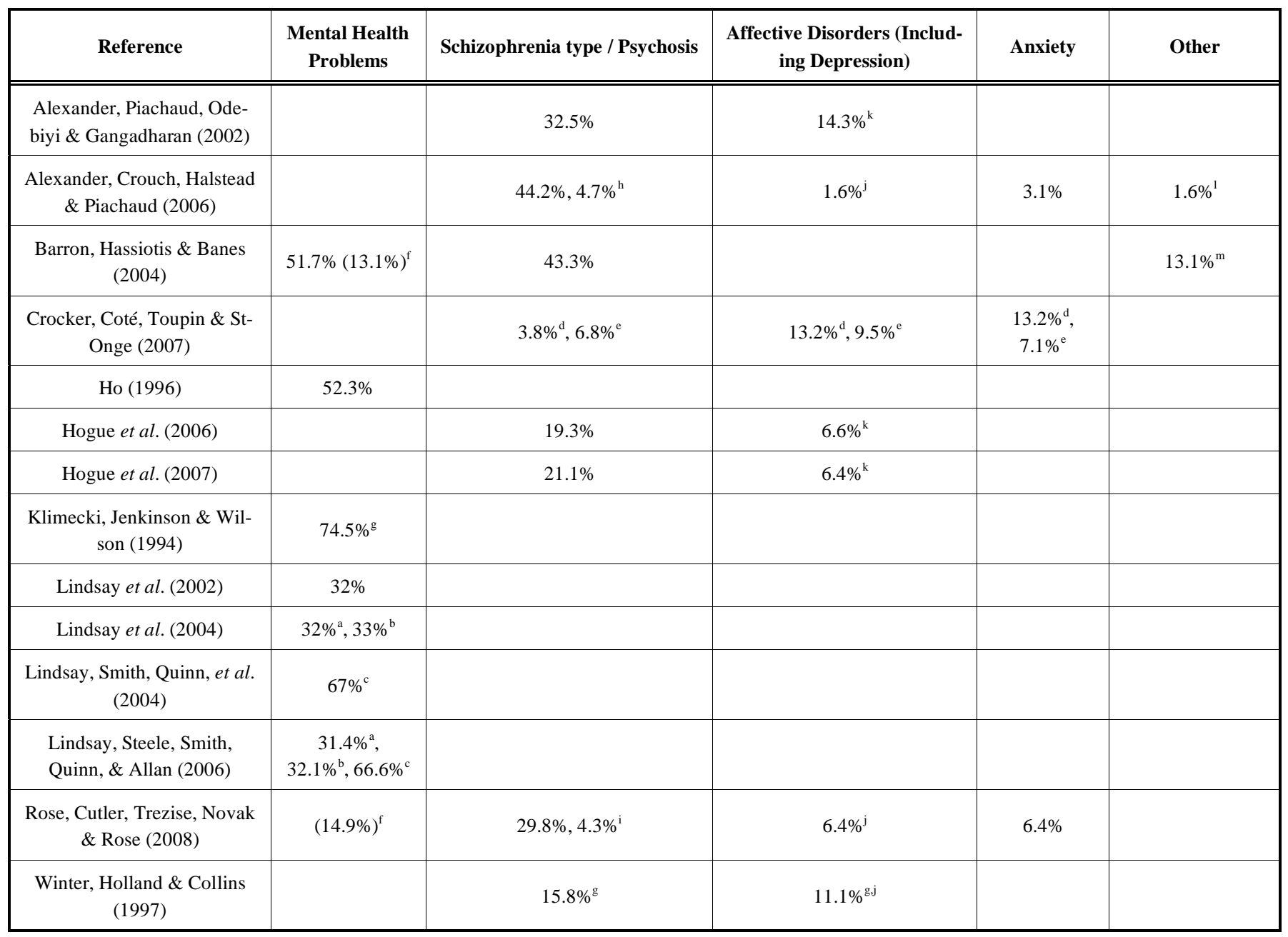

${ }^{\mathrm{a}}$ Sex Offenders, ${ }^{\mathrm{b}}$ Non-Sex Offenders, ${ }^{\mathrm{c}} \mathrm{Female}$ Offenders, ${ }^{\mathrm{d}}$ Probable ID group, ${ }^{\mathrm{e}}$ Borderline ID group. ${ }^{\mathrm{f}}$ Co-morbid disorders, ${ }^{\mathrm{g}}$ Self reported prior psychiatric history, ${ }^{\mathrm{h}}$ Schizoaffective disorder, ${ }^{\mathrm{i}}$ Manic Depressive Psychosis, ${ }^{\mathrm{j}}$ Depression, ${ }^{\mathrm{k}}$ Affective Disorder, ${ }^{\mathrm{l}}$ Obsessive Compulsive Disorder, ${ }^{\mathrm{m}}$ Autistic Symptoms.

chologist within 48 hours to confirm diagnosis. The percentage of participants diagnosed with a mental health problem was $30.2 \%$ and $23.4 \%$ for the ID and borderline groups respectively. This data was not reported in the paper but supplied by the authors in response to a request for further information. Crocker et al. (2007) [14] comparison group of detainees without an ID were also diagnosed with a similar rate of mental health problems $(29.0 \%)$. Although around $20 \%$ of participants were intoxicated at the time of assessment, Crocker et al. (2007) [14] found no significant differences in the composition of the ID, borderline and non-ID groups when intoxicated individuals were excluded from the analysis, indicating that intoxication did not affect the group constitution. In this study the assessment of mental health appeared to be relatively thorough, which may account for the lower rate of mental health problems identified.

\section{COMMUNITY SAMPLES}

Barron, Hassiotis and Banes (2004) [15] examined two groups of people with ID who offended in a community setting; those who were known to ID services and those who were known to forensic mental health services. Barron et al. (2004) [15] screened probation services, community mental health teams and elsewhere in non-ID agencies for individuals with an ID. Following assessment using the WAIS-R the group of individuals not known to ID services had a significantly greater IQ than the group known to ID services (69.7 vs. 63.5), although no range was reported for either group. Social impairment was questioned during the screening process but there was no formal assessment of participant's functional abilities. Barron et al. (2004) [15] used a broad definition of offending behavior to reflect the overlap of offending and challenging behaviors. They found no significant difference in the rates of mental health problems between the two groups, with a total rate of $51.7 \%$, although the group of individuals not known to ID services was small (17).

Rose et al. (2008) [11] reported on a sample of 47 individuals who used community ID services, the sample consisted of 42 males and 5 females. Individuals were in receipt of ID services and would have met the service criteria for having an ID. Two people $(4.3 \%)$ of the sample had a borderline ID, the majority of the sample had a mild ID (53\%), and the remainder either had a more severe disability or had no recorded level of ID $(17 \%)$. It is not reported what the range of intellectual functioning was. The study used a broad definition for offending to include 'offending-like behaviors' 
to include individuals who would have been diverted from the criminal justice system as a result of their ID. The study used informant based interviews and case notes to collect the data. Around $46 \%$ of the sample had been diagnosed with a mental health problem at some time over the previous 5 years.

\section{INPATIENT SETTINGS}

Alexander et al. (2002) [9] examined case referrals to a medium secure unit in the UK. The sample size was 79, IQ or adaptive behavior was clinically assessed by a least two multi-disciplinary professionals. The sample included some individual without ID (9.2\%), although the majority had either a borderline ID $(36.8 \%)$ or a mild ID $(44.7 \%)$, with some having moderate $(7.9 \%)$ or a severe ID (1.3\%). However, they state that some individuals who were assessed as having a normal IQ or adaptive functioning scores were considered by clinicians as having a borderline ID. Around $33 \%$ of patients did not have an index offence nor were they involved with the criminal justice system. In this study about $47 \%$ had affective and psychotic disorders and $58.4 \%$ of referrals had a personality disorder, this latter figure is more than double that found in any other studies, which be may a product of the specialized nature of this service.

Alexander, Crouch, Halstead and Piachaud (2006) [16] conducted a case file review and follow-up of individuals with ID who had been referred, and subsequently discharged from a medium secure unit. There is a slight overlap in data between this study and Alexander et al. (2002) [9] although this study includes only those who were admitted following referral. The sample size was 64, although for the purpose of the study the sample was divided into subgroups depending on the time at which they were discharged from the unit. Individuals assessed as having normal IQ or adaptive functioning scores but assessed by clinicians as having below normal intelligence were categorized as having a borderline ID. Overall, the study group contained one (1.6\%) individual with normal intelligence, $17(26.6 \%)$ with a borderline ID, $35(54.7 \%)$ with a mild ID and $11(17.1 \%)$ with a moderate ID. Individuals discharged earlier (1987 - 1993) were more likely to have a borderline ID or normal intelligence than those discharged later (1994 - 2000), though the characteristics of mental health problems between the groups was similar. The clinical diagnosis of mental health problems was recorded for individuals at the time of discharge, but it does not state how this diagnosis was made. Alexander et al., (2006) [16] recorded the rates of re-offending and relapse of mental health problems, as well as those requiring readmission to hospital. However, patients with a diagnosis of schizophrenia were four times less likely to reoffend (defined as having contact with the police in this case). Considering the specialist nature of this service this sample may not be representative of people with an ID who offend.

\section{MIXED SETTING SAMPLES}

Hogue et al. (2006) [17] also includes data reported by Hogue et al. (2007) [18] which excluded some individuals from the previous study due methodological issues. Hogue et al. (2006) [17] included samples from three different sites; high security hospital (73), medium and low security hospital (70) and a community-based sample (69). The mean reported IQ of the total sample was $66.0(\mathrm{SD}=8.61)$, IQ scores were taken from case files following clinical assessment and IQ testing. The majority of the total sample had been diagnosed with "mental retardation" as described by ICD $10(82.5 \%)$. Information regarding mental health problems was taken from case files. Individuals were diagnosed with psychotic disorder $(19.3 \%)$ and affective disorder $(6.6 \%)$ according to ICD 10 criteria. The rates of psychotic disorder varied significantly between sites, with the medium and low secure hospital having the lowest rate $(8.6 \%)$, the community sample $(20.3 \%)$ and the high security hospital having the highest rate $(28.8 \%)$. There was some overlap between participants who had at one point used a service then been transferred to another site. This sample may not be representative of people in the UK with an ID who offend, as the high security hospital is the only one of its kind in the UK.

Hogue et al. (2007) [18] used the same methodology and the same population, yet used the Emotional Problem Scale [19] to assess at psychological, emotional and behavioral problems in 171 of the 212 study sample. The mean IQ of the study group was 65.88 which did not differ significantly between the high security hospital, medium and low security hospital and a community-based sample, however the range of IQ scores is not reported. As a result the rates of psychotic disorder $(21.1 \%)$ and affective disorder (6.4\%) differ from Hogue et al. (2006) [17].

Lindsay et al. (2006) [20] is an update of referrals of three other papers: Lindsay et al. (2002) [21], Lindsay et al. (2004) [22] and Lindsay, Smith, Quinn et al. (2004) [23]. As a result of this only the most recent article will be considered here. Lindsay et al. (2006) used a sample from a community service with associated inpatient units which consisted of female offenders (21), male sexual offenders (121) and male non-sexual offenders (105). There was no significant difference in IQ scores between the sex offences group (64.9), non-sex offences group (65.4) and female group (67.5). The majority of individuals fell into the mild ID range, with around a quarter having a borderline ID and $3-5 \%$ in the range of moderate ID. Rates for mental health problems were only reported for major disorders (schizophrenia, bipolar disorder or major depression) however female offenders were recorded as having more than twice the incidence of mental health problems $(66.6 \%)$ than males $(31.4 \%$ and $32.1 \%)$.

\section{METHODOLOGICAL ISSUES}

All of these papers report considerable variation in the rates of mental health problems in people with an ID who offend. One major issue with these papers is the group of individuals included within the surveys. While all of the papers suggest that they are reviewing people with an Intellectually Disability, on careful examination none of them strictly adhere to current definitions of ID such as that defined by the British Psychological Society (2001) [4]. Most definitions would include a significant impairment of intellectual functioning (IQ of 69 or less); a significant impairment of adaptive/social functioning and; an age of onset before adulthood of impairments. Using this definition would exclude a number of participants in all of the studies. For example, only Ho (1996) [10] formally assessed adaptive behavior. Similarly, only Ho (1996) [10] makes any mention 
Table 4. The Number of Male and Female Participants in the Reviewed Articles

\begin{tabular}{|c|c|c|}
\hline Reference & Number of Females in Study Group (\%) & Number of Males in Study Group (\%) \\
\hline $\begin{array}{l}\text { Alexander, Piachaud, Odebiyi \& Gangadharan } \\
\text { (2002) }\end{array}$ & $15(19)$ & $64(81)$ \\
\hline Alexander, Crouch, Halstead \& Piachaud (2006) & $15(23)$ & $49(77)$ \\
\hline Barron, Hassiotis \& Banes (2004) & $8(13)$ & $53(87)$ \\
\hline Ho (1996) & $23(8)$ & $259(92)$ \\
\hline Hogue et al. (2006) & & $212(100)$ \\
\hline Hogue et al. (2007) & & $171(100)$ \\
\hline Kilmecki, Jenkinson \& Wilson (1994) & & $75(100)$ \\
\hline Lindsay, Smith, Quinn, et al. (2004) & $18(100)$ & \\
\hline Lindsay, Steele, Smith, Quinn, \& Allan (2006) & $21(9)$ & $226(91)$ \\
\hline Rose, Cutler, Trevise, Novak \& Rose (2008) & $5(11)$ & $42(89)$ \\
\hline Winter, Holland \& Collins (1997) & $1(5)$ & $20(95)$ \\
\hline
\end{tabular}

${ }^{\mathrm{a}}$ ID Group and Borderline ID Group ${ }^{\mathrm{b}}$ Sex Offenders.

of the age of onset of disability and reported that approximately $34 \%$ of their sample had sustained a prior head injury as a result of vehicle accidents or complications during birth.

Sampling methods may also have caused a bias and skew to the selection of samples for inclusion in studies. Samples from high security hospitals and medium secure units may not be representative of many people with an ID who offend. McBrien (2003) [24] has suggested that the self-report method, employed in Winter et al. (1997) [13] and Barron et al. (2004) [15], may be over-inclusive and increase the numbers of individuals with a borderline ID in these studies. Differences between criminal justice systems may also influence the composition of samples with individuals with an ID being diverted to a variety of specialist ID services at different stages in the judicial system depending on the country's criminal justice system. These differences also influence the definition of offence used within the studies with some studies including individuals who had not been convicted as they had been diverted from the criminal justice system due to their ID (e.g. Rose et al, 2008) [11]. Variations in these procedures are likely to influence the composition of samples in the different papers reviewed.

The differences in the rates of mental health problems recorded may also be due to the variation in diagnostic practice and the flexible use of terms such as 'mental illness' and 'psychiatric illness'. Klimecki et al. (1994) [12] reported that $74.5 \%$ of individuals in their sample had a prior psychiatric history. The term psychiatric history covers a wide range of illnesses and disorders, and having a psychiatric history is much more inclusive than having a current diagnosis. Similarly a number of studies only reported an overall rate of mental health problems in terms of major disorders (schizophrenia, bipolar disorder or major depression). This could provide a more restricted view at the prevalence of mental health problems. Crocker et al. (2007) [14] seemed to take a more thorough approach to the screening for mental health problems by using a standardized psychiatric instrument and re-assessment by a psychologist within 48 hours. This may have resulted in the lower prevalence rate (around 30\%) in this study. Considering the relatively high rates of mental health problems, it is perhaps surprising that few studies explicitly state the level of co-morbidity of mental disorders. Barron et al. (2004) [15] reports a prevalence of $13.1 \%$ for individuals scoring on one or more diagnostic criteria for mental health problems, however this also includes a number of individuals with autistic symptoms. Rose et al. (2008) [11] found that $14.9 \%$ of their study group had more than one "concern", which included a range of mental health problems, personality disorder, autistic symptoms, physical health problems and drug/alcohol misuse. The lack of information regarding co-morbidity certainly represents a problem in the majority of the reported studies, as rates of separate mental health problems are recorded it is not clear what the overall number of individuals with mental health problems is in some samples.

The high proportion of males with an ID who offend found in the reviewed articles is generally similar to those of other studies $[1,25]$ and in the people without an ID who offend [26]. In the only analysis in a group of female offenders Lindsay et al. (2006) [20] found over twice the rate of mental health problems found in a male sample. Although other studies (See Table 4) do include females with an ID who offend they do not report gender differences in terms of the rates of mental health problems probably due to the small numbers of females included. Maden, Swinton and Gunn (1994) [27] reported that females who offend were more 
likely to have a psychiatric diagnosis then males (57\% vs. $38 \%$ respectively). This suggests it may be particularly important to treat mental health problems in women offenders.

\section{DISCUSSION}

Rates of mental health problems in people with an ID who offend in the studies reviewed here vary from $26 \%$ [17] up to $74.5 \%$ [12]. This appears to be higher than in people with an ID who do not offend, however the variations in methodology, sample selection and assessment strategies make it difficult to obtain a clear overall picture.

Estimates of the prevalence of mental health problems amongst general populations of adults with ID also vary greatly. Studies have reported rates of mental health problems of between $14 \%$ and $40 \%$ [28, 29]. Considering that the majority of people with an ID who offend appear to have a mild, moderate or borderline intellectual disability, the literature relating to these individuals warrants special attention. Deb et al. (2001) [28] found that $14.4 \%$ of people with a mild ID had mental health problems.

Different definitions of mental health problems produce different results. Klimecki et al. (1994) [12] reported that $74.5 \%$ of ID offenders had a "psychiatric history", while Lindsay et al. (2004) [22] found that around 32\% of male offenders had "mental health problems". Alexander et al. (2002) [9] commented that the rate of mental health problems, personality disorders and other issues found would make them a particularly difficult group to manage.

\section{SCHIZOPHRENIA/PSYCHOSIS/SCHIZOAFFECTIVE DISORDER}

Schizophrenia and psychotic disorders appear to be the most prevalent single mental health problem reported in studies of people with ID who offend. Rates vary from around $4 \%$ up to $44 \%$ (Table 3). Only one study (Rose et al., 2008) [11] reports a rate of manic depressive psychosis at $4.3 \%$ of the sample. Alexander et al. (2006) [16] reports a rate of schizoaffective disorder at $4.7 \%$. These diagnoses may have been included in the schizophrenia categories in other studies, and different classification systems may also account for the range in rates.

In a review of literature on mental health problems in adults with an ID, Whitaker (2006) [30] reported rates of schizophrenia from $1 \%$ up to $5.1 \%$. However, other studies have found higher rates particularly in those with a mild or moderate ID. Holden and Gitlesen (2004) [31] reported that $15 \%$ of individuals with a moderate ID had psychosis compared to no individuals with a severe or profound ID in their study group. Reiss (1982) [32] reported prevalence rates of $17 \%$ and $47 \%$ for individuals with a mild/moderate ID and a severe ID respectively. The majority of people with an ID who offend fall into the mild/moderate ID category and this group appears to have a higher rate of psychotic disorders than the people with an ID who do not offend. This may be due to the nature of the populations studied and the variety in diagnostic strategies used in the studies. For example, when individuals enter the criminal justice setting they may be more thoroughly assessed by health professionals which may lead more effective diagnosis. The effects of co morbid psychotic difficulties and offending in people with an ID is clearly complex and worthy of further investigation.

\section{AFFECTIVE DISORDERS (INCLUDING ANXIETY AND DEPRESSION)}

Affective disorders including anxiety and depression appear to be the second most common mental health problem reported in people with ID who offend. Rates of affective disorder vary from around $7 \%$ up to $13 \%$ (Table 3). In terms of depression, rates vary from $1.6 \%$ up to $11 \%$ (Table 3). Anxiety disorders are sometimes reported separately. The rate of depression in people with an ID has been reported to be between $1 \%$ to $21 \%$ [[31, 33]. However, other studies have found that severity of disability is related to the rate of depression seen in adults with an ID. Reiss (1982) [32] found that $20 \%$ of individuals with a mild/moderate ID had depression compared to no individuals with a severe ID. Similarly, Holden \& Gitlesen (2004) [31] reported that $21 \%$ of people with a moderate ID were depressed compared to $2 \%$ of people with a severe or profound ID. Only three studies present data on the prevalence of anxiety disorders in people with ID who offend. These ranged between 3.1 and $13.2 \%$. Alexander et al. (2006) [16] also report a rate of obsessive compulsive disorder (OCD) of $1.6 \%$.

Estimates of the prevalence of anxiety disorders in people with an ID who do not offend vary considerably. Rojahn, Borthwick-Duffy and Jacobson (1993) [34] found that only $0.3 \%$ had an anxiety disorder, in contrast, Reiss (1990) [35] reported a rate of $31.4 \%$ for anxiety "symptoms". Holden \& Gitlesen (2004) [31] reported that $48 \%$ of individuals with a moderate ID and $13 \%$ of individuals with a severe or profound ID had an anxiety disorder. This variation makes any comparisons difficult to interpret.

\section{CONCLUSION}

It is clear that a significant proportion of people with an ID who offend have a diagnosed mental health problem. However, the variation in methodologies and samples used to collect this data makes it difficult to draw any firm conclusions about prevalence rates. Mental health problems, particularly schizophrenia and psychotic disorders appear to be higher in people with an ID who offend. More research is required that systematically evaluates both offending and non-offending populations using similar methodologies before a definitive conclusion can be reached. If mental health problems exist in any individual who offends they may be a factor in the offending process. Mental health problems may also need to be treated effectively in order to rehabilitate individuals. Glaser and Florio (2004) [8] suggest that mental health "may be making a contribution, in its own right, to the behaviours and difficulties which lead to offending by an intellectually disabled person" (p. 592). People with an ID and a mental health problem tend to exhibit challenging behaviours which may increase the likelihood of contact with the criminal justice system $[2,7]$. Having an unmet mental health need may increase the risk of offending due to a reduced ability to cope with living in the community. Future research needs to focus on the prevalence of mental health problems and the influence of mental health problems on offending, treatment and recidivism, to develop a greater insight on the role of mental health in the lives of these individuals. 


\section{REFERENCES}

[1] Holland T, Clare I, Mukhopadhyay T. Prevalence of "criminal offending" by men and women with intellectual disability and the characteristics of "offenders": Implications for research and service development. J Intellect Disabil Res 46(1): 6-20 (2002).

[2] Simpson MK, Hogg J. Patterns of offending among people with intellectual disability: A systematic review. Part 2: predisposing factors. J Intellect Disabil Res 45: 397-406 (2001).

[3] Simpson J, Martin M. Green J. (2001). The Framework Report. Sydney: NSW Council for Intellectual Disability.

[4] British Psychological Society. (2001). Learning Disability: Definitions and Contexts. Leicester: B.P.S. Professional Affairs Board.

[5] Hatton C. (1998) Intellectual Disabilities-epiddemology and causes. In Emerson E, Hatton C, Bromley J, Caine, A. Eds. Clinical psychology and intellectual disabilities, 1998 (pp. 20-38). Chichester: Wiley.

[6] Lindsay WR. Research and literature on sex offenders with intellectual and developmental disabilities. J Intellect Disabil Res 46: 74-85 (2002).

[7] Chan J, Hudson C, Sigafoos J. A preliminary study of offenders with intellectual disability and psychiatric disorder. Ment Health Aspects Dev Disabil 6: 147-152 (2003).

[8] Glaser W, Florio D. Beyond specialist programmes: A study of the needs of offenders with intellectual disability requiring psychiatric attention. J Intellect Disabil Res 48(6): 591-602 (2004).

[9] Alexander RT, Piachaud J, Odebiyi L, Gangadharan SK. Referrals to a forensic service in the psychiatry of learning disability. Br J Forensic Pract 4: 29-33 (2002).

[10] Ho T. Assessment of retardation among mentally retarded criminal offenders: An examination of racial disparity. J Crim Justice 24: 337-350 (1996).

[11] Rose JL, Cutler C, Trezise K, Novak D, Rose D. Individuals with an intellectual disability who offend. Br J Dev Disabil 106: 19-30 (2008).

[12] Klimecki MR, Jenkinson J, Wilson L. A study of recidivism among offenders with an intellectual disability. Aust NZ J Dev Disabil 19: 204-219 (1994).

[13] Winter N, Holland AJ, Collins S. Factors predisposing to suspected offending by adults with self-reported learning disabilities. Psychol Med 27: 595-607 (1997).

[14] Crocker AG, Coté G, Toupin J, St-Onge B. Rate and characteristics of men with an intellectual disability in pre-trial detention. J Intellect Dev Disabil, 32(2): 143-152 (2007).

[15] Barron P, Hassiotis A, Banes J. Offenders with intellectual disability: A prospective comparative study. J Intellect Disabil Res 48: 69-76 (2004).

[16] Alexander RT, Crouch K, Halstead S, Piachaud J. Long-term outcome from a medium secure service for people with intellectual disability. J Intellect Disabil Res 50: 305-315 (2006).

[17] Hogue TE, Steptoe L, Taylor J, et al. A comparison of offenders with intellectual disability across three levels of security. Crim Behav Ment Health 16: 13-28 (2006).

[18] Hogue TE, Mooney P, Morrissey C, et al. Emotional and behaviorual problems in offenders with intellectual disability: Comparative data from three forensic services. J Intellect Disabil Res 51(10): 778-785 (2007)
[19] Prout HT, Strohmer DC. (1991). Emotional Problems Scales. Professional manual for the behaviour rating scales and the self-report inventory. Lutz, FL: Psychological Assessment Resources Inc.

[20] Lindsay WR, Steele L, Smith AHW, Quinn K, Allan R. A community forensic intellectual disability service: Twelve year follow up of referrals, analysis of referral patterns and assessment of harm reduction. Legal Crim Psychol 11: 113-130 (2006).

[21] Lindsay WR, Smith AHW, Law J, et al. A treatment service for sex offenders and abusers with intellectual disability: Characteristics of referrals and evaluation. J App Res Intellect Disabil 15: 166-174 (2002).

[22] Lindsay WR, Smith AHW, Law J, et al. Sexual and non-sexual offenders with intellectual and learning disabilities: A comparison of characteristics, referral patterns and outcome. J Interpersonal Violence 19: 875-890 (2004).

[23] Lindsay WR, Smith AHW, Quinn K, et al. Women with intellectual disability who have offended: Characteristics and outcome. J Intellect Disabil Res 48: 580-590 (2004).

[24] McBrien J. The intellectually disabled offender: Methodological problems in identification. J App Res Intellect Disabil 16: 95-106 (2003).

[25] Noble JHJ, Conley RW. Toward an epidemiology of relevant attributes. In RW Conley, R Luckasson, GN Bouthilet, Eds. The criminal justice system and mental retardation: Defendants and victims, 1992 (pp. 17-53). Baltimore, MD: Paul H. Brookes Publishing.

[26] Marshall T, Simpson S, Stevens A. (2000). Health Care in Prisons: A Health Care Needs Assessment. Birmingham: University of Birmingham.

[27] Maden AM, Swinton P, Gunn J. A criminological and psychiatric survey of women serving a prison sentence. Br J Crim 34: 172-191 (1994).

[28] Deb S, Thomas M, Bright C. Mental disorder in adults with intellectual disability. 1: Prevalence of functional psychiatric illness among a community based sample population aged between 16 and 64 years. J Intellect Disabil Res 45: 495-505 (2001).

[29] Rojahn J, Tasse' MJ. Psychopathology in mental retardation. In JW Jacobson, JA Mulick, Eds. Manual of diagnosis and professional practice in mental retardation 1996 (pp. 147-156). Washington, DC: American Psychological Association.

[30] Whitaker S. Read S. The prevalence of psychiatric disorders among people with intellectual disabilities: An analysis of the literature. J App Res Intellect Disabil 19: 330-345 (2006).

[31] Holden BI, Gitlesen JP. The association between severity of intellectual disability and psychiatric symptomatology. J Intellect Disabil Res 48: 556-562 (2004).

[32] Reiss S. Psychopathology and mental retardation: survey of a developmental disabilities mental health program. Ment Retard 20: 128-132 (1982).

[33] Cooper SA, Collacott R. Mania and Down's syndrome. Br J Psychiatry 162: 739-743 (1993)

[34] Rojahn J, Borthwick-Duffy SA, Jacobson JW. The association between psychiatric diagnosis and severe behaviour problems in mental retardation. Ann Clin Psychiatry 5: 163-170 (1993).

[35] Reiss S. Prevalence of dual diagnosis in community-based day programs in the Chicago metropolitan area. Am J Ment Retard 94 578-585 (1990).

() Hobson and J. L. Rose; Licensee Bentham Open.

This is an open access article distributed under the terms of the Creative Commons Attribution License (http://creativecommons.org/licenses/by/2.5/), which permits unrestrictive use, distribution, and reproduction in any medium, provided the original work is properly cited. 